\title{
Scale dependence of the beta diversity-scale relationship
}

\author{
Y. Zhang ${ }^{1}$, K. Ma1,4, M. Anand ${ }^{2}$, W. Ye ${ }^{3}$ and B. Fu ${ }^{1}$ \\ I State Key Laboratory of Urban and Regional Ecology, Research Center for Eco-Environmental Sciences, Chinese \\ Academy of Sciences, Beijing, 100085, China \\ 2 Global Ecological Change Laboratory, School of Environmental Sciences, University of Guelph, Guelph, Ontario, \\ N1G 2W1, Canada \\ ${ }^{3}$ Key Laboratory of Vegetation Restoration and Management of Degraded Ecosystems, South China Botanical Garden, \\ Chinese Academy of Sciences, Guangzhou, 510650, China \\ ${ }_{4}^{4}$ Corresponding author.Tel/Fax: 86-10-62849104,Email:mkm@rcees.ac.cn
}

Keywords: Alpha diversity, Diversity partitioning, Gamma diversity, Power law, Scaling.

\begin{abstract}
Alpha, beta, and gamma diversity are three fundamental biodiversity components in ecology, but most studies focus only on the scale issues of the alpha or gamma diversity component. The beta diversity component, which incorporates both alpha and gamma diversity components, is ideal for studying scale issues of diversity. We explore the scale dependency of beta diversity and scale relationship, both theoretically as well as by application to actual data sets. Our results showed that a power law exists for beta diversity-area (spatial grain or spatial extent) relationships, and that the parameters of the power law are dependent on the grain and extent for which the data are defined. Coarse grain size generates a steeper slope (scaling exponent $z$ ) with lower values of intercept $(c)$, while a larger extent results in a reverse trend in both parameters. We also found that, for a given grain (with varying extent) or a given extent (with varying grain) the two parameters are themselves related by power laws. These findings are important because they are the first to simultaneously relate the various components of scale and diversity in a unified manner.
\end{abstract}

Abbreviations: DBH-Diameter at Breast Height, SAR-Species-area relationship.

\section{Introduction}

Ecologists have long distinguished three fundamental components of species diversity: alpha diversity $(\alpha)$, which measures the species diversity at the local scale, beta diversity $(\beta)$, which measures the extent of local diversity change in a region, and gamma diversity $(\gamma)$, which measures the total diversity of a region (Whittaker 1972). All of these diversity components are scale dependent: alpha diversity depends on the size of the local scale, gamma diversity depends on the size of the regional scale, and beta diversity, which links alpha and gamma diversity, depends on both local and regional scales (Loreau 2000). However, considerable attention has been given primarily to the scale dependency issues of alpha (or gamma) diversity.

The species-area relationship (SAR) is well-known for describing the scale dependency behavior of alpha or gamma diversity components when measured by species richness (the species richness and diversity are use synonymously in this paper). The most common form of SAR is the power law scaling relationship ( $S=c A^{z}$, where $S$ is number of species or species richness; $A$ is area measured; parameter $z$ is the scaling exponent with value less than 1 , and parameter $c$ is a constant, Arrhenius 1921). This can help determine the minimal sampling area for biodiversity surveys, extrapolate species richness in response to habitat loss, and plan optimal sizes for nature reserves (He and Legendre 1996, Dengler 2009). Actually, the power law scaling relationship of SAR is also dependent on scale, as it has been shown that its parameters (especially the scaling exponent $z$ ) vary with spatial scale (Crawley and Harral 2001, Drakare et al. 2006).

Spatial scale can be separated into several components, including grain, extent, and lag (Wiens 1989), but grain and extent are used primarily for SAR scale dependence studies. Grain is the size of the individual units of observation - the quadrats of a field ecologist or the sampling units of a statistician-which correspond to the minimum scale sampled (often the size of individual quadrats) in a study, and extent is the overall area encompassed by a study (Wiens 1989). Palmer and White (1994) observed that spatial grain and extent influence the shape of the SAR curve in a plant community. Crawley and Harral (2001) considered the spatial extent effects on slope of the power SAR of plant communities, and found that scaling exponent $z$ has a unimodal pattern which shows the peak at intermediate spatial extent sizes with low values at small and large spatial extent size. Drakare et al. (2006) considered the effects of both spatial grain and spatial extent on SARs and provided evidence of the fact that coarse spatial grain follows a steeper slope $(z)$, while spatial extent does not show clear pattern on scaling exponent $z$. The inconsistency between these two works on the spatial extent effect on the SAR is due partially to the fact that the results 
of Drakare et al. (2006) were based on 794 SARs from different geographical location and taxonomic groups which also impact on the slope of SAR. Drakare et al. (2006) found that steeper slope of SAR are generated at lower latitudes and by larger organisms also. The scale dependency of SAR is still an open issue, especially for the extent effects. To our knowledge, there have been surprisingly few attempts to quantify both spatial extent and grain dependence of the power law SAR in one dataset analytically and systematically. Spatial grain and extent exist simultaneously for any dataset. In the SAR, the minimum area $\left(A_{m i n}\right)$ sampled for analysis corresponds to the spatial grain, and the maximum area $\left(A_{\max }\right)$ sampled for analysis corresponds to the spatial extent which made the spatial grain and spatial extent have been included in an implicit way. Therefore, it is hard to explore the scale (spatial grain and spatial extent) dependency of SAR analytically and systematically in an explicit way.

Beta diversity, which is considered as important as alpha diversity in understanding ecosystem functions and management and biodiversity conservation (Wilson and Shmida 1984; Legendre et al. 2005), has the potential to include the spatial grain and extent explicitly when dealing with scale dependency issues. Although diverse definitions and measures for beta diversity have been reviewed, discussed, and recommended extensively (see papers of Wilson and Schmida 1984, Vellend 2001, Koleff et al. 2003, Tuomisto 2010a, 2010b, Anderson et al. 2011), the beta diversity in our work refers to that which is related directly to alpha and gamma diversity components. It is well known that two different perspectives exist for relating beta diversity components directly with alpha and gamma diversity components: the multiplicative perspective (beta ${ }_{m u l}=$ gamma/alpha-1; Whittaker 1972) and the additive perspective (beta ${ }_{\text {add }}=$ gamma-alpha; MacArthur et al. 1966). There have been extensive discussions with respect to which perspective is better in partitioning an "independent" beta diversity (Jost 2007, Ricotta and Marignani 2007, Ricotta 2008, de Bello et al. 2010, Chao et al. 2012, c.f. a recent Forum in Ecology [2010] edited by Ellison, and papers in it). However, there is no perfect metric for measuring beta diversity (Ricotta 2010) and both additive and multiplicative partitioning can be extremely useful in studies focusing on species diversity (Veech and Crist 2010). Kiflawi and Spencer (2004) found that the perspectives for partitioning beta diversity are directly related to each other (beta ${ }_{m u l}=$ beta $_{\text {add }} /$ alpha). For either decomposition approach, beta diversity is determined by alpha diversity, which corresponds to spatial grain, and gamma diversity, which corresponds to spatial extent; this means that beta diversity explicitly includes spatial grain and extent simultaneously, hence lending itself naturally to the study of its scale dependency issues.

However, it is surprising that few works explore the scale dependency of beta diversity. Recently, Barton el al. (2013) proposed a framework for spatial scaling of beta diversity, but case studies on quantification of the scaling relationship between beta diversity and area are needed. As beta diversity includes both spatial grain and extent, Barton et al. (2013) suggested that three ways could been used in exploring the beta diversity and area scaling relationship: 1) fixing grain while varying extent, 2) fixing extent while varying grain, and 3 ) co-varying extent and grain. The main purpose of our work is to explore whether scale dependence properties exist for scaling relationship between beta diversity and scale (spatial extent and spatial grain). Specifically, we want to analyze the spatial grain effects on the scaling relationship between beta diversity and spatial extent and the spatial extent effects on the scaling relationship between beta diversity and spatial grain. Therefore, the first two ways suggested by Barton et al. (2013) for spatial scaling of beta diversity were used in our work. The scaling relationship between beta diversity and scale is assumed in power law form. This assumption is based on the fact that alpha diversity and gamma diversity could be scaling up by power law. As previously stated that beta diversity is a function of alpha diversity and gamma diversity, when exploring beta diversity and extent scaling relationship, namely fixing alpha diversity but changing gamma diversity, and given the power law scaling of gamma diversity, it is reasonable to assume beta and extent may have power law scaling relationship. Similarly, when exploring beta diversity and grain scaling relationship, namely fixing gamma diversity but changing alpha diversity, and given the power law scaling of alpha diversity, it is reasonable to assume beta and extent may have power law scaling relationship also. Based on this power law scaling relationship assumption between beta diversity and scale, we explored the scale dependency of this relationship by the mathematical inference. The power law beta diversity scaling assumption and mathematical predictions on the scale dependency of this scaling relationship were also verified through the analysis of forest community data from an elevational transect of warm, temperate deciduous broadleaf oak (Quercus liaotungensis Koidz) forest in Beijing, North China and a 20-ha subtropical, evergreen broadleaf forest plot in Guangdong province, South China.

\section{Methods}

\section{Mathematical inference}

We first assumed that beta diversity is related to spatial grain or extent in a power law form and then demonstrated through mathematical inference the scale dependence of these power law relationships. For this purpose, we introduced mathematical formulations to express changing extent $(E)$ and grain $(G)$ : Let $\gamma[i]$ be the number of species for extent size $E[i]$, where $i=[1,2, \ldots, \mathrm{n}]$ represents a series of increasing extent sizes, such that $E[i+1]>E[i]$ and $\gamma[i+1]>\gamma[i]$. Similarly, let $\bar{\alpha}[j]$ (where $\bar{\alpha}[j]<\gamma[i]$ ) be the average species number for grain size $G[j](j=1,2, \ldots, \mathrm{m}$, represents a series of increasing grain sizes), such that $G[j+1]>G[j]$ and $\bar{\alpha}[j+1]>\bar{\alpha}[j]$. Through straightforward reinterpretations of the power law SAR, the relationship between $\gamma$ and spatial extent, and between $\bar{\alpha}$ and spatial grain are both in the form of a power law when diversity is measured using species richness [but see Zhang et al. (2006) for a demonstration that this extends to most diversity indices (e.g., Shannon, Simpson)]. 
Beta diversity and spatial scale: the power-law scaling relationship

We used two directly correlated beta diversity measures from the multiplicative and additive perspectives of diversity partitioning to explore the beta diversity and area scaling relationship. For the additive perspective, the beta diversity can be measured by:

$$
\beta_{\text {add }}=\gamma-\bar{\alpha}
$$

For the multiplicative perspective, we used the modified beta diversity measure to detect the "full changes" among local sites (Whittaker 1972):

$\beta_{m u l}=\gamma / \bar{\alpha}-1$

Because these measures are related directly with $\beta_{\text {mul }} \beta_{\text {add }} /$ $\bar{\alpha}$ (Kiflawi \& Spencer 2004), we only present the mathematical inference and data tests of additive approach for beta diversity $\left(\beta_{\text {add }}\right)$ (the results of beta diversity $\left(\beta_{m u l}\right)$ partitioned by multiplicative approach were provide in Supplemental Materials), but our criticism applies to both.

As suggested by Barton et al. (2013), the beta diversity and area scaling relationship can be explored either by fixing spatial grain, but changing spatial extent or fixing spatial extent, but changing spatial grain. This means that the beta diversity and area relationship can be expressed by the beta diversity and spatial extent relationship or the beta diversity and spatial grain relationship. For the former, the relationship between beta diversity and extent takes the following form:

$\beta_{\text {add }}=c E[i]^{z}-\bar{\alpha}$

where $c E[i]^{z}$ is $\gamma$ at extent size $E[i]$ when gamma diversity and spatial extent have a power law scaling relationship. We assumed that the beta diversity and spatial extent curve also could be described by a power law curve also:

$\beta_{\text {add }} \propto c_{\text {add }-e} E[i]^{z_{\text {add }-e}}$

where $c_{\text {add-e }}$ is the intercept and $z_{\text {add-e }}$ is the scaling exponent of power law beta diversity and spatial extent relationship respectively. For the latter, the relationship between beta diversity and grain takes the following form:

$\beta_{\text {add }}=\gamma-c G[j]^{z}$

where $c G[j]^{z}$ is $\bar{\alpha}$ at grain size $G[j]$ when alpha diversity and spatial grain have a power law scaling relationship. We also assumed that the relationship between beta diversity and spatial grain could be described by a power law curve:

$\beta_{\text {add }} \propto c_{\text {add }-g} G[j]^{-z_{\text {add }-g}}$

where $c_{\text {add-g }}$ is the intercept and $-z_{\text {add-g }}$ is the scaling exponent of power law beta diversity and spatial extent relationship respectively. As the scaling exponent is negative, we only using the absolute value to compare the extent effects on the slope of this power law scaling relationship.

\section{Scale dependence of power-law beta diversity and spatial} scaling relationships

The scale dependence of the scaling relationship between beta diversity and area (spatial grain or extent) are based on the assumed equations above. We began with the observation that the linear form of equation (3) and (5) can be generalized as:

$$
\log \left(y_{k}\right)=\log (c)+z \log \left(x_{k}\right)
$$

where $y_{k}$ represents beta diversity $\left(\beta_{a d d}\right), x_{k}$ represents spatial scale (spatial extent $E[i]$ or spatial grain $G[j])(k=i$ when dealing with beta diversity and extent relationship, $k=j$ for beta diversity and grain relationship), $z$ and $c$ correspond to the scaling exponent $\left(z_{\text {add-e }},-z_{\text {add-g }}\right)$ and intercept $\left(c_{\text {add-e }}, c_{\text {add-g }}\right)$, respectively in equation (4) and (6). According to equation (6), $z$ and $c$ can be simplified as:

$z=\frac{\log \left(y_{k+1}\right)-\log \left(y_{k}\right)}{\log \left(x_{k+1}\right)-\log \left(x_{k}\right)}$

and

$\log (c)=\log \left(y_{k}\right)-z \log \left(x_{k}\right)$

Equation (9) can be written as:

$c=y_{k} x_{k}^{-z}$

Therefore, the scaling exponents $\left(z_{\text {add-e }},-z_{\text {add-g }}\right)$ and intercepts $\left(c_{\text {add-e }}, c_{\text {add-g }}\right)$ can be quantified by formulas (8), (10). Now, we will show the grain scale dependence of the power law beta diversity and extent relationship and the extent scale dependence of power law beta diversity and grain relationship.

\section{Scale dependence of power law beta diversity and extent relationship}

In this case, the grain size $G[j]$ will change to determine the behavior of the beta diversity and spatial extent relationship in formula (4). The scaling exponent is determined by substituting the additive beta diversity $\beta_{\text {add }}=\gamma[i]-\bar{\alpha}[j]$ and extent $E[i]$ into formula (8):

$$
\begin{aligned}
& z_{\text {add }-e}[j]=\log \left(1+\frac{\gamma[i+1]-\gamma[i]}{\gamma[i]-\bar{\alpha}[j]}\right)\left(\frac{1}{\log (E[i+1])-\log (E[i])}\right) \\
& z_{\text {add }-e}[j+1]=\log \left(1+\frac{\gamma[i+1]-\gamma[i]}{\gamma[i]-\bar{\alpha}[j+1]}\right)\left(\frac{1}{\log (E[i+1])-\log (E[i])}\right)
\end{aligned}
$$

where $z_{\text {add-e }}[j]$ and $z_{\text {add-e }}[j+1]$ are the scaling exponent of power law additive beta diversity and extent relationship at grain size $G[j]$ and $G[j+1]$, respectively. Comparing the right part of formula (11) and (12) for all i and j, because $\bar{\alpha}[j+1]>\bar{\alpha}$ $[j], \gamma[i+1]>\gamma[i], \gamma[i]>\bar{\alpha}[j]$,we can confirm that $z_{\text {add-e }}[j+1]>$ $z_{\text {add-e }}[j]$.

The corresponding intercepts is determined by substituting scaling exponent $z_{\text {add-e }}$, extent $E[i]$ and the multiplicative beta diversity $\beta_{\text {add }}=\gamma[i]-\bar{\alpha}[j]$ into formula (10):

$$
c_{\text {add }-e}[j]=(\gamma[i]-\bar{\alpha}[j]) E[i]^{-z_{\text {add }-e}[j]}
$$

$c_{\text {add }-e}[j+1]=(\gamma[i]-\bar{\alpha}[j+1]) E[i]^{-z_{\text {add }-e}[j+1]}$

where $c_{\text {add-e }}[j]$ and $c_{\text {add-e }}[j+1]$ are the intercept of power law additive beta diversity and extent relationship at grain size 
$G[j]$ and $G[j+1]$ respectively. Comparing the right part of formula (13) and (14), and because $\bar{\alpha}[j+1]>\bar{\alpha}[j], \gamma[i]>\bar{\alpha}[j]$, $z_{\text {add-e }}[j+1]>z_{\text {add-e }}[j]$, we can conclude that $c_{\text {add-e }}[j+1]<c_{\text {add-e }}[j]$.

\section{Scale dependence of power law beta diversity and grain relationship}

In this case, the extent size $E[i]$ will change to determine the behavior of the beta diversity and spatial grain relationship in formula (6). The scaling exponent is determined by substituting the additive beta diversity $\beta_{a d d}=\gamma[i]-\bar{\alpha}[j]$ and grain $G[j]$ into formula (8):

$z_{\text {add-g }}[i]=-\log \left(1-\frac{\bar{\alpha}[j+1]-\bar{\alpha}[j]}{\gamma[i]-\bar{\alpha}[j]}\right)\left(\frac{1}{\log (G[j+1])-\log (G[j])}\right)$

$z_{\text {add- } g}[i+1]=-\log \left(1-\frac{\bar{\alpha}[j+1]-\bar{\alpha}[j]}{\gamma[i+1]-\bar{\alpha}[j]}\right)\left(\frac{1}{\log (G[j+1])-\log (G[j])}\right)$

where $z_{\text {add-g }}[i]$ and $z_{\text {add-g }}[i+1]$ are the scaling exponent of power law additive beta diversity and grain relationship at extent size $E[i]$ and $E[i+1]$, respectively. Comparing the right part of formula (15) and (16) for all $i$ and $j$, and because $\bar{\alpha}$ $[j+1]>\bar{\alpha}[j], \gamma[i+1]>\gamma[i], \gamma[i]>\bar{\alpha}[j]$,we can confirm that $z_{\text {add-g }}[i+1]<z_{\text {add-g }}[i]$.

The corresponding intercept is determined by sub-

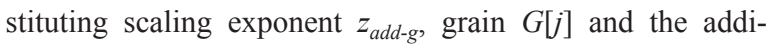
tive beta diversity $\beta_{\text {add }}=\gamma[i]-\bar{\alpha}[j]$ into formula (10):

$$
\left.c_{\text {add -g }}[i]=(\gamma[i]-\bar{\alpha}[j]) \times G[j]^{z_{\text {add }-\mathrm{g}}[i]}\right)
$$

$c_{\text {add }-g}[i+1]=(\gamma[i+1]-\bar{\alpha}[j]) \times G[j]^{z_{a d d-g}^{[i+1]}}$

where $c_{\text {add-g }}[i]$ and $c_{\text {add-g }}[i+1]$ are the intercept of power law additive beta diversity and grain relationship at extent size $E[i]$ and $E[i+1]$ respectively. When $G[j]=1, c_{\text {add-g }}[i]=\gamma[i]$ $-\bar{\alpha}[j], c_{\text {add-g }}[i+1]=\gamma[i+1]-\bar{\alpha}[j]$, and because $\gamma[i+1]>\gamma[i]$, we can conclude that $c_{\text {add-g }}[i+1]>c_{\text {add-g }}[i]$.

According to predictions above, for the power law relationship between beta diversity $\left(\beta_{\text {add }}\right)$ and extent, we can predict that, for a given system, large grain size will result in a larger slope $z\left(z_{\text {add-g }}\right)$ and smaller intercept $c\left(c_{\text {add-g }}\right)$. In contrast, a smaller extent size will result in steeper slope $z$ $\left(z_{\text {add-e }}\right)$ and smaller intercept $c\left(c_{\text {add-e }}\right)$ for the power law beta diversity $\left(\beta_{\text {add }}\right)$ and grain relationship.

\section{Application}

We tested our assumptions and analytical results with two data tests. First, we verified that the relationship between beta diversity and scale [either $E$ or $G$; formula (4) and (6)]) has a power law form. Next, we verified our theoretical predictions of the scale dependency of the power law beta diversity and that of the scale relationship. For the spatial grain dependence, we plotted the power law scaling exponent $\left(z_{\text {add-e }}\right)$ and intercept $\left(c_{\text {add-e }}, c_{\text {add-g }}\right)$, which were determined by the first data tests, against grain size, respectively. For the spatial ex- tent dependence, we plotted the power law scaling exponents $\left(z_{\text {add-g }}\right)$ and intercepts $\left(c_{\text {add-g }}\right)$ against extent size. The power law was selected for data fitting.

\section{Data sets}

The first dataset was an elevational transect (1000$1770 \mathrm{~m})$ of warm-temperate deciduous broadleaf Oak $(Q$. liaotungensis Koidz) forest in Beijing, North China. The data were collected in the Donglingshan Mountains, 100 $\mathrm{km}$ northwest of Beijing City, in 2003, hereinafter referred to as the DLS transect. Ten transects were established from the foot to the top of every western slope in the study area to compose a continuous altitude gradient $(1020 \mathrm{~m}-1770 \mathrm{~m})$ that overlapped the distribution range of $Q$. liaotungensis in the study area completely. The width of each transect is $10 \mathrm{~m}$, and the length range of each transect is from $80 \mathrm{~m}$ to $200 \mathrm{~m}$, depending on the altitude range and slope degree of each slope. We however ignored the insignificant slope and slope degree effects on the spatial distribution of species, so all transects merged into a single transect 1190 meters long and consisting of 119 quadrats, each $10 \mathrm{~m} \times 10 \mathrm{~m}$. In each quadrat, all woody species (trees and shrubs) and herb species were identified. Fifty-three families, 137 genera, and 251 species were recorded (See Zhang et al. 2006 for more details on this data set). The second dataset was a 20 ha $(500 \mathrm{~m} \times 400$ $\mathrm{m})$ permanent subtropical, evergreen broadleaf forest plot in Guangdong province, South China. The plot was established in the Dinghushan reserve in November 2004, hereinafter referred to as the DHS plot. The survey consisted of enumerating all free standing trees and shrubs at least $1 \mathrm{~cm}$ in diameter at breast height $(\mathrm{DBH})$, positioning each one by geographic coordinates on a reference map and identifying them by species. Fifty-six families, 119 genera, 210 species and 71617 individuals with DBH $>1 \mathrm{~cm}$ were recorded in this dataset (See Li et al. 2009, for more details on this data set). For both datasets, we used presence/absence data only.

\section{Beta diversity calculation}

For the DLS transect dataset, we selected 960m (altitude range between $1110 \mathrm{~m}$ to $1730 \mathrm{~m}$ ) in length for calculation of beta diversity. This is convenient in partitioning the transect with grain size $10 \mathrm{~m} \times 10 \mathrm{~m}, 20 \mathrm{~m} \times 10 \mathrm{~m}, 30 \mathrm{~m} \times 10 \mathrm{~m}, 40$ $\mathrm{m} \times 10 \mathrm{~m}$, and $60 \mathrm{~m} \times 10 \mathrm{~m}$. For example, the grain size in $10 \mathrm{~m} \times 10 \mathrm{~m}$ encompassed size ranges from $20 \mathrm{~m} \times 10$ $\mathrm{m}$ (minimum value) to $960 \mathrm{~m} \times 10 \mathrm{~m}$ (maximum value) as the transect was $960 \mathrm{~m}$ in length. All possible samples for each extent size were analyzed, resulting in 95 samples with a minimum extent size of $20 \mathrm{~m} \times 10 \mathrm{~m}, 94$ samples for extent size of $30 \mathrm{~m} \times 10 \mathrm{~m}$, and so on, ending in 1 sample for extent size of $960 \mathrm{~m} \times 10 \mathrm{~m}$. The same process was completed for the four other grain sizes. We then examined the grain effects of the power law beta diversity and its relationship to extent. We selected seven extent sizes $(240 \mathrm{~m} \times 10 \mathrm{~m}, 360 \mathrm{~m} \times 10 \mathrm{~m}$, $480 \mathrm{~m} \times 10 \mathrm{~m}, 600 \mathrm{~m} \times 10 \mathrm{~m}, 720 \mathrm{~m} \times 10 \mathrm{~m}, 840 \mathrm{~m} \times 10 \mathrm{~m}$, $960 \mathrm{~m} \times 10 \mathrm{~m}$ ) to analyze the extent effects of the power law 
beta diversity and grain relationships. Woody species (trees and shrubs) and herb species were analyzed separately.

For the DHS plot data set, we selected a subplot size of $480 \mathrm{~m} \times 360 \mathrm{~m}$ to calculate beta diversity of the woody species. This is convenient in partitioning the plot with grain size $10 \mathrm{~m} \times 10 \mathrm{~m}, 20 \mathrm{~m} \times 20 \mathrm{~m}, 30 \mathrm{~m} \times 30 \mathrm{~m}, 40 \mathrm{~m} \times 40 \mathrm{~m}$, and $60 \mathrm{~m} \times 60 \mathrm{~m}$. We also calculated beta diversity for all possible samples for all potential extent sizes, as described previously. The five grain sizes selected were used for grain effect analysis. For the extent effect analysis, we selected seven extent sizes $(120 \mathrm{~m} \times 240 \mathrm{~m}, 120 \mathrm{~m} \times 360 \mathrm{~m}, 240 \mathrm{~m} \times 240 \mathrm{~m}$ (and $120 \mathrm{~m} \times 480 \mathrm{~m}), 240 \mathrm{~m} \times 360 \mathrm{~m}, 240 \mathrm{~m} \times 480 \mathrm{~m}, 360 \mathrm{~m} \times$ $360 \mathrm{~m}, 360 \mathrm{~m} \times 480 \mathrm{~m})$

\section{Data fitting}

The power laws in biology data could be fitted by linear regression on log-transformed data or nonlinear regression directly. Which one is selected for power law fitting should be based on a combination of biological plausibility and analysis of the error distribution (Xiao et al. 2011). We used the method developed by Xiao et al. (2011) to judge which way is used for the parameter estimation of our data and find that our data are suitable for using nonlinear regression method for power law fitting. So the nonlinear regression was used for power law parameter estimation (Ritz and Streibig 2008). All calculation and data fitting work were taken under $\mathrm{R}$ package (R Development Core Team, 2011).

\section{Results}

Beta diversity and extent showed a power law relationship for all data sets for all grain sizes [Fig. 1 (a), (b), (c)], two grain sizes were selected as examples for each data]. Beta diversity and extent size were related positively. The power law relationship also existed for the beta diversity and grain relationship under varying spatial extent size [Fig. 1 (d), (e),
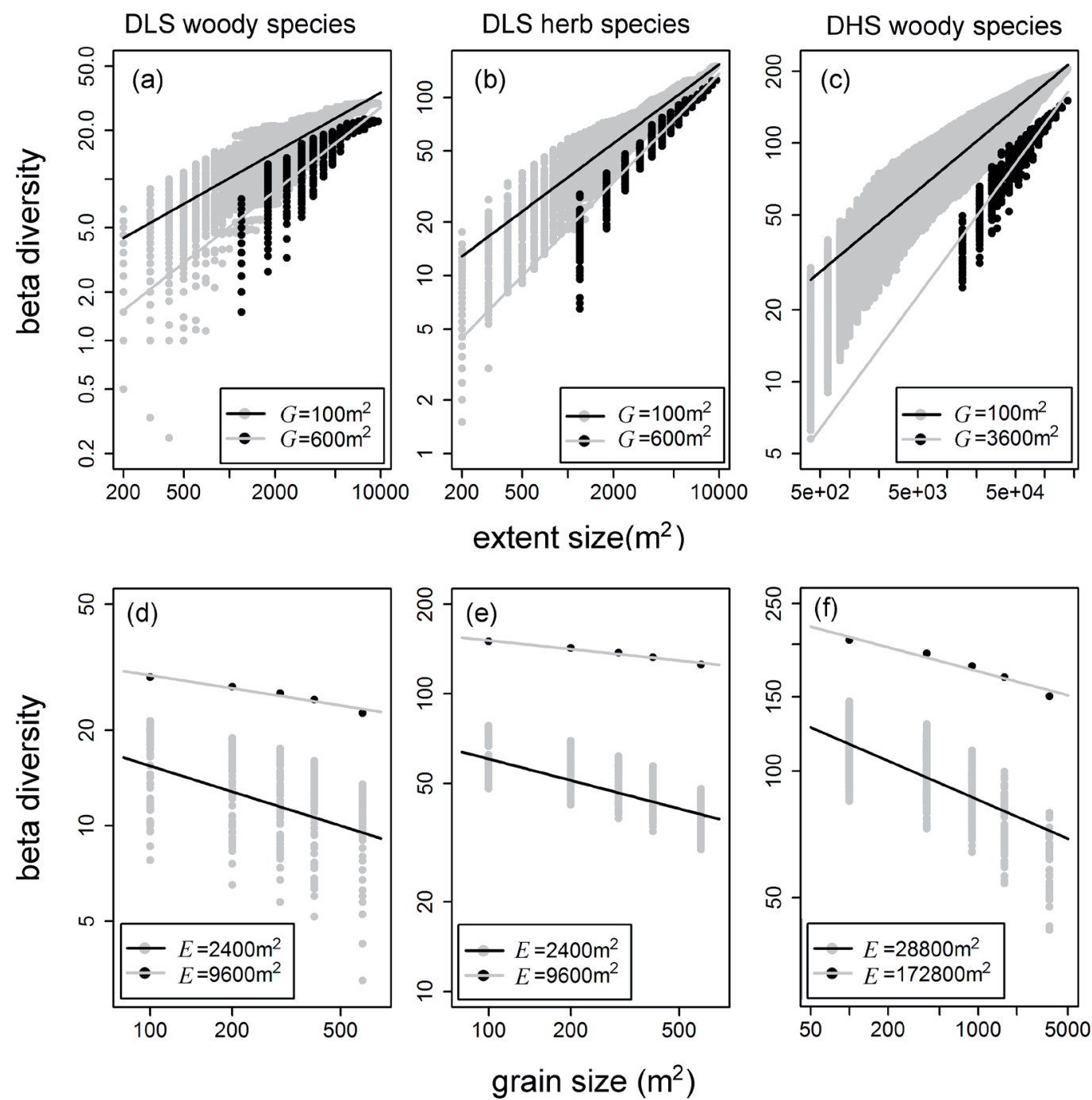

Figure 1. Examples of beta diversity and scale relationship plots using the power law for data fitting. (a), (b), and (c) show the beta diversity and spatial extent (e) relationship for two selected grain sizes (f). Figures (d), (e), and (f) show the beta diversity and spatial grain relationship for two selected extent sizes. Logarithmic scale was used for each axis of the plots. 
(f)], two extent sizes were selected as examples for each data also]. In contrast to the beta diversity and extent relationship, beta diversity was correlated negatively with grain size.

The intercept $c$ and scaling exponent $z$ of the beta diversity and extent power law relationship and grain size had power law relationship (Fig. 2). Larger grain size had smaller intercept $c$, but steeper scaling exponent $z$. The intercept $c$ and scaling exponent $z$ of the beta diversity and grain power law relationship and grain size also had power law relationship (Fig. 3). In contrast to the grain effect on the scaling relationship of beta diversity and extent, small extent size had smaller intercept $c$, but steeper scaling exponent $z$.

\section{Discussion}

The power law relationship between the number of species, the alpha or gamma diversity component, and scale is one of the oldest and best-documented patterns in ecology (Arrhenius 1921), and is considered as one of the few robust laws of ecology (Lawton 1999, Lomolino 2000, Martín and
Goldenfeld 2006). It is easy to predict that increasing spatial extent and fixing spatial grain will increase beta diversity, and increasing spatial grain while fixing spatial extent will decrease beta diversity (c.f. the expected trend proposed by Barton et al. (2013) for beta diversity scaling), this is due to the fact that a larger spatial extent will increase environmental heterogeneity in space which in turn increase beta diversity and coarse grain size for sampling will miss fine-scale environmental heterogeneity (MacNally et al. 2004) which in turn decrease beta diversity. Our work firstly verified that, in our datasets, the beta diversity component and scale had a power law relationship. However, the most intriguing result in our work is that the power law beta diversity and scale (spatial grain or spatial extent) relationship is itself scale dependent, especially on the results that the parameters of the power law beta diversity and scale in our data sets showed power law relationship with scale.

Our work predicted and verified that coarse grain size produces a steeper slope of power law beta diversity and spatial extent relationship. The power law beta diversity and extent relationship in our work is analogy to power law SAR,
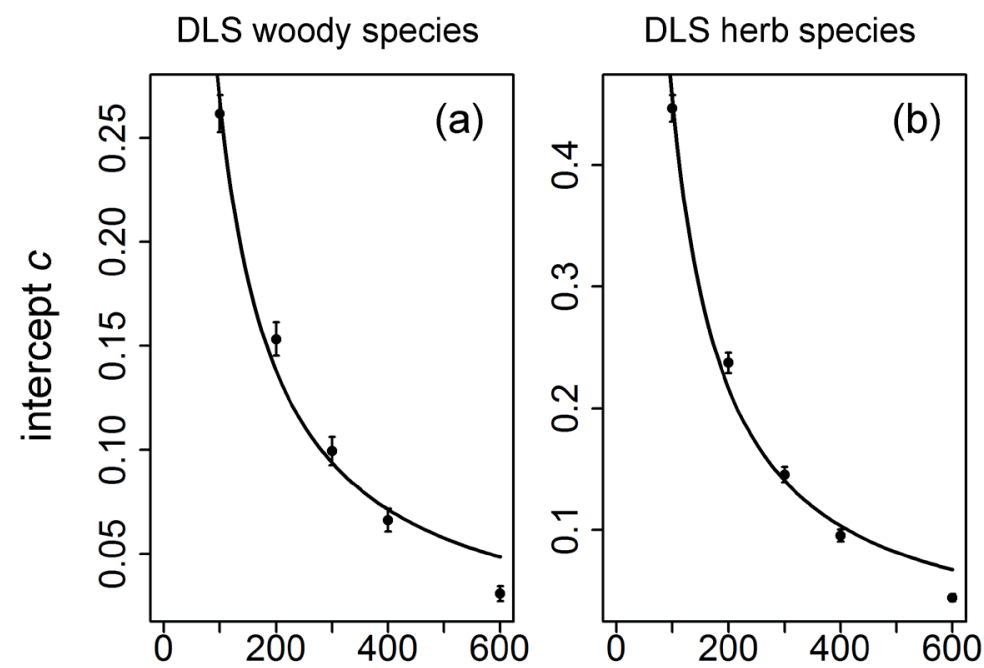

\section{DHS woody species}
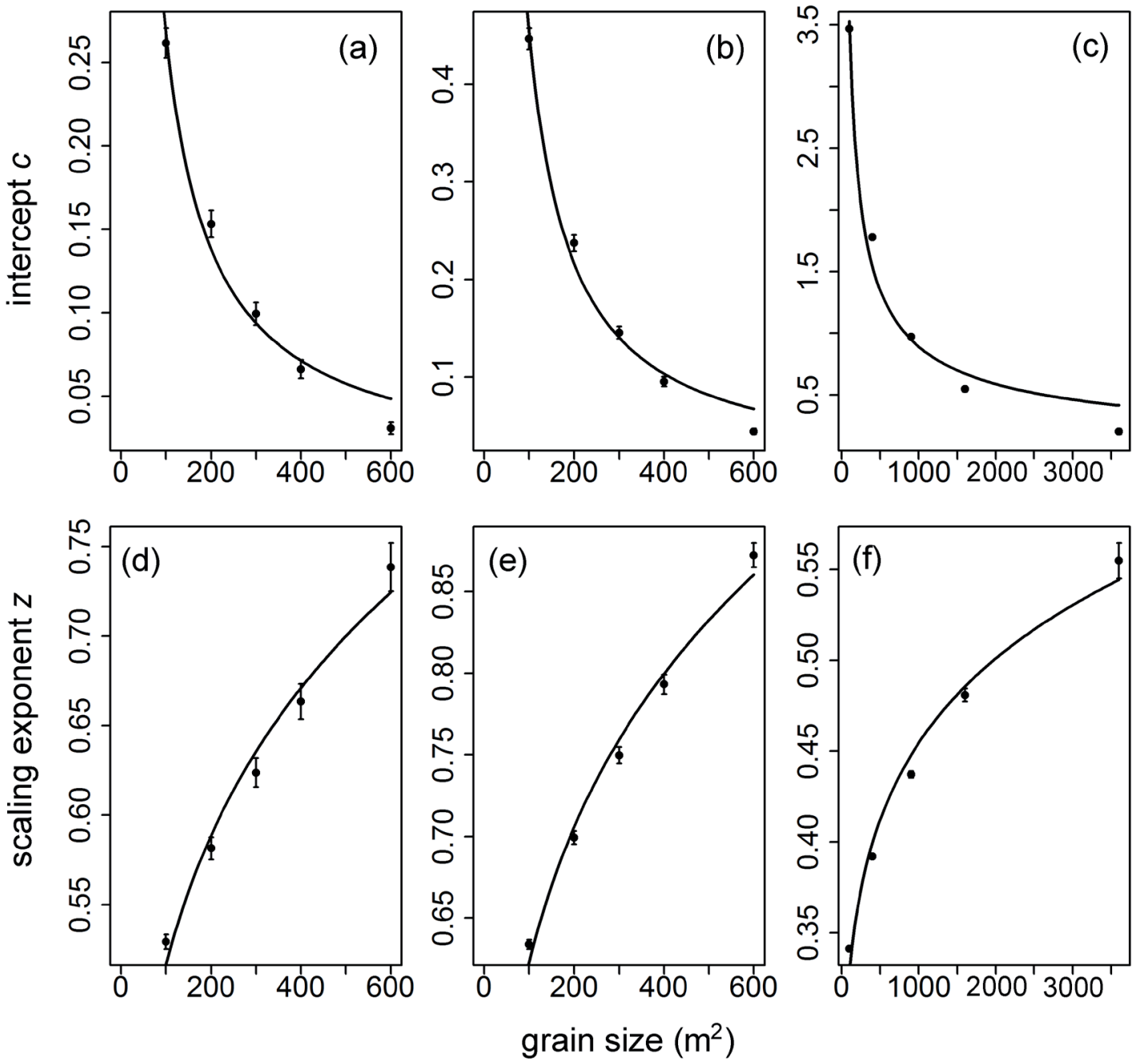

Figure 2. Scale dependency of the intercept $c$ and slope $z$ of the power law beta diversity and spatial extent relationship. Power law was used to fit the relationship between the parameters (intercept $c$ or slope $z$ ) and spatial grain relationship. 
but they differed in that the former explicitly includes grain while the later includes the grain in an implicit way. In SAR studies, the theoretical works (Rosenzweig 1995, Hubbell 2001) and meta-analysis (Drakare et al. 2006) also found that large grain size have steeper slopes. However, in our work, smaller extent size will have steeper slope of the power law beta diversity and grain relationship. The extent effects on the slope of SAR are not consistent in previous studies. Crawley and Harral (2001) showed that the intermediate spatial extent size produces the steepest slope of SAR. While in the work of Drakare et al. (2006), the slope of SAR does not show any trend with spatial extent size. Turner and Tjørve (2005) suggested that habitat heterogeneity, sampling method and taxonomic differences, which ultimately effect the scale dependence of SAR, this might, in part, explain the difference between the results of Drakare et al. (2006) and those of Crawley and Harral (2001) on the extent effects of SAR. In our work, the data sets differed in habitat heterogeneity, sampling methods and taxonomic, but the scale dependency pattern of the slope $z$ (and intercept $c$ ) of power law beta diversity and scale relationship all consistent with our theoreti- cal predictions. This implies that the trend of the parameters showed in our work might be the general pattern for describing the scale dependency of power law beta diversity and scale relationship; thus, further tests on it are recommended

Our data sets also verified the predictions of our theoretical analysis that parameter $c$ varies with scale. This parameter has been virtually ignored as one deserving biological or statistical explanation in SAR studies. MacArthur and Wilson (1967) considered it solely as a fitted constant relating to local environmental conditions. Connor and McCoy (1979), however, argued that this parameter represents the possible species richness when a sample of one unit of area is examined. We believe both views are important, because local environmental conditions will determine the possible value of species richness per unit of area. The parameter $c$ in our work represents the possible value of beta diversity in one unit of scale (grain or extent), which can reflect the environmental conditions of the scale examined. If the extent is considered, then in the beta diversity and spatial extent relationship, a larger grain would cause the loss of environmental heterogeneity at smaller grain size and decrease the value of
DLS woody species
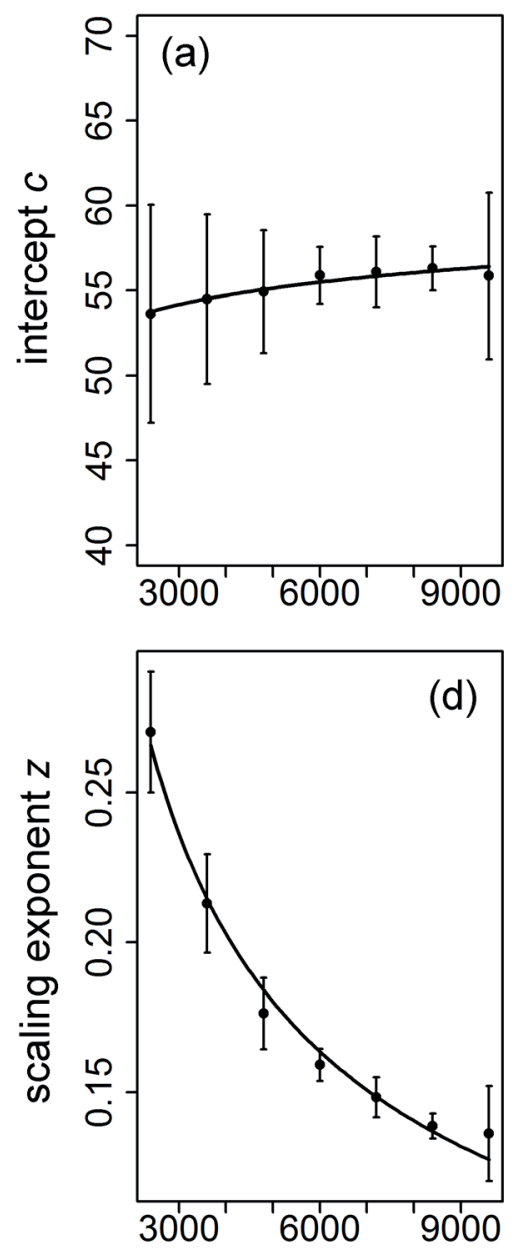

DLS herb species
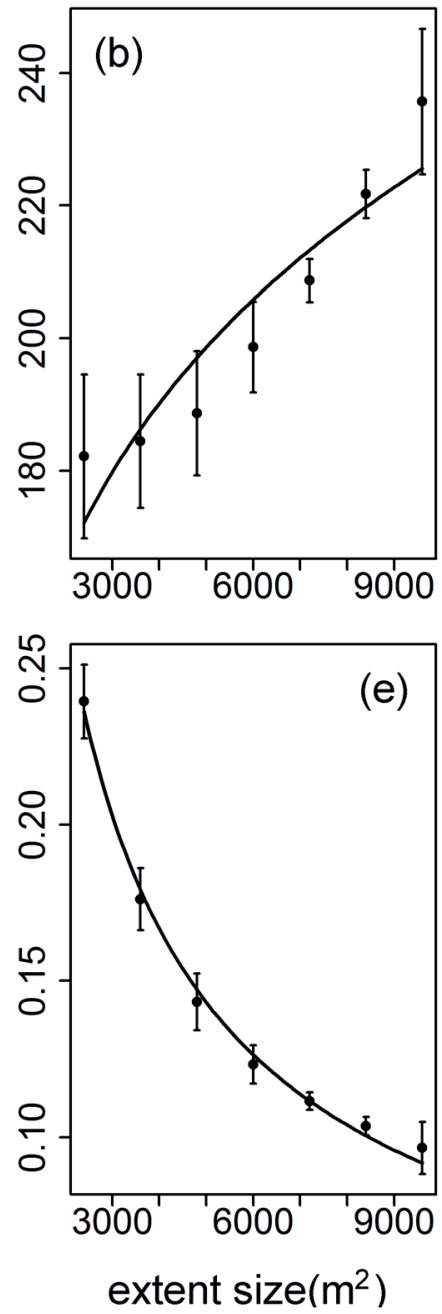

DHS woody species
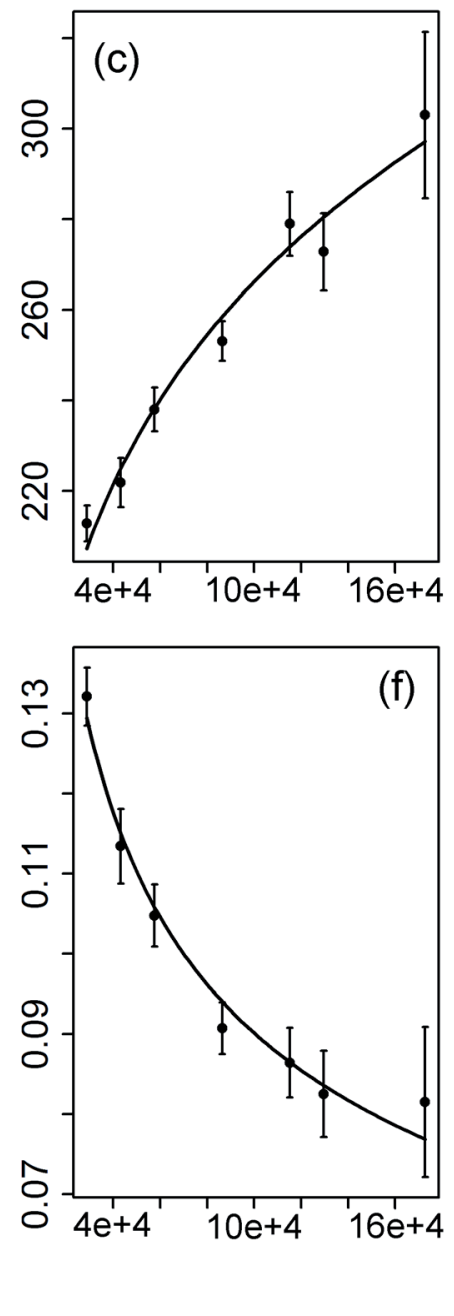

Figure 3. Scale dependency of the intercept $c$ and slope $z$ of the power law beta diversity and spatial grain relationship. Power law was used to fit the relationship between the parameters (intercept $c$ or slope $z$ ) and spatial extent relationship. 
beta diversity, thereby decreasing $c$ as well. Similarly, for the beta diversity and spatial grain relationship, a larger extent increases environmental heterogeneity and increases the value of beta diversity; hence, $c$ will increase.

Connor and McCoy (1979) suggested that the power law SAR is purely a sampling phenomenon and that values of parameters must be considered simple fitted constants devoid of biological meaning. While our work does not pretend to unravel any particular ecological process, we showed that scaledetermined patterns should be closely tied to spatial scale, which is a lurking factor (Sandel and Smith 2009). We also found that alpha, beta, and gamma diversity relationships all exist within a unifying scaling framework, which has been suggested as the greatest challenge to ecologists (Loreau 2000). The beta diversity and spatial extent relationship reflects the fact that changing beta diversity is comparable to gamma diversity change under a constant alpha diversity, while the beta diversity and spatial grain relationship reflects the fact that changing beta diversity is comparable to alpha diversity change under a specified gamma diversity. In our work, alpha diversity fully determines beta diversity for a given extent, while gamma diversity fully determines beta diversity for a given grain. Similarly, the scale dependence of the beta diversity and scale relationship implies that the relationship between alpha, beta and gamma diversity may also be scale dependent.

Scale occupies a central role in ecological research, as it links patterns on the one hand and process on the other (Levin 1992). Therefore, considering how results vary as a function of scale is a necessary first step to putting our knowledge regarding patterns and processes into perspective (Rahbek 2005). Using beta diversity to study scale effects is ideal, as it incorporates two important aspects of spatial scale (grain and extent). Our work shows the role of spatial scale on beta diversity in two ways: we verified that a power law scaling relationship exists for beta diversity, and we found that this scaling relationship is itself scale dependent with a power law form. For the former, it provides a quantitative way for beta diversity extrapolation (either up scaling or down scaling). Theoretically, any SAR curve could be used in beta diversity scaling. He and Legendre (1996) found that the curves in describing SAR are also dependent on scale. Our work only consider the most frequent used power law curve, but other curves are suggested to be used as alternatives when exploring beta diversity scaling at variable scales. For the later, it makes the scale dependency of power law beta diversity scaling relationship predictable. This allows us to put into theoretical context varying observations of scale-dependent behavior of beta diversity observed in empirical studies and gives a more precise form to the scale dependence of power law beta diversity-area relationships. However, the scale dependency property of beta diversity scaling also arise the issue on the selection of spatial scale for the accurate beta diversity extrapolation, cause any small deviation to the proper scale for extrapolation will cause large overestimation or underestimation. More attention had focused on the curve selection for proper extrapolation of diversity (He and Legendre 1996, Scheiner 2003, Whittaker and Matthews 2014). Our work highlights the importance of developing new method and designing new experiment on the selection of proper scale for diversity extrapolation in future.

Acknowledgements. This research is supported by National Key Basic Research Program (2009CB421104), National Natural Science Foundation of China (30870459), and the Innovation Project of the State Key Laboratory of Urban and Regional Ecology of China (SKLURE2013-1-02). M.A. thanks the Natural Science and Engineering Council of Canada, the Canada Research Chairs program and the Canadian Foundation for Innovation for financial support for this research collaboration. We thank M. Tonge for her valuable comments.

\section{References}

Anderson, M.J., T.O. Crist, J.M. Chase, M. Vellend, B.D. Inouye, A.L. Freestone, N.J. Sanders, H.V. Cornell, L.S Comita, K.F. Davies, S.P. Harrison, N.J.B. Kraft, J.C. Stegen and N.G. Swenson. 2011. Navigating the multiple meanings of beta diversity: a roadmap for the practicing ecologist. Ecol. Lett. 14: 19-28.

Arrhenius, O. 1921. Species and area. J. Ecol. 9: 95-99.

Barton, P.S., S.A. Cunningham, A.D. Manning, H. Gibb, D.B. Lindenmayer and R.K. Didham. 2013. The spatial scaling of beta diversity. Global Ecol. Biogeogr. 22: 639-647.

Chao A., C. Chiu and C.H. Hsieh. 2012. Proposing a resolution to debates on diversity partitioning. Ecology 93: 2037-2051.

Connor, E.F. and E.D. McCoy.1979. The statistics and biology of the species-area relationship. Am. Nat. 113: 791-833.

Crawley, M.J. and J.E. Harral. 2001. Scale dependence in plant biodiversity. Science 291: 864-868.

de Bello, F., S. Lavergne, C. N. Meynard, J. Lepš and W. Thuiller. 2010. The partitioning of diversity: showing Theseus a way out of the labyrinth. J. Veg. Sci. 21: 992-1000.

Dengler, J. 2009. Which function describes the species-area relationship best? A review and empirical evaluation. J. Biogeogr. 36: 728-744.

Drakare S., J.J. Lennon and H. Hillebrand. 2006. The imprint of the geographical, evolutionary and ecological context on speciesarea relationships. Ecol. Lett. 9: 215-227.

Ellison, A.M. 2010. Partitioning diversity. Ecology 91: 1962-1963.

He, F.L. and P. Legendre. 1996. On species-area relations. Am. Nat. 148: 719-737.

Hubbell, S.P. 2001. The Unified Neutral Theory of Biodiversity and Biogeography. Princeton University Press, Princeton, NJ.

Jost, L. 2010. Independence of alpha and beta diversities. Ecology 91: 1969-1974.

Kiflawi M. and M. Spencer. 2004. Confidence intervals and hypothesis testing for beta diversity. Ecology 85: 2895-2900.

Koleff, P., K.J. Gaston and J.J. Lennon. 2003. Measuring beta diversity for presence-absence data. J. Anim. Ecol. 72: 367-382.

Lawton, J.H. 1999. Are there general laws in ecology? Oikos 84: 177192.

Legendre, P., D. Borcard and P.R. Peres-Neto. 2005. Analyzing beta diversity: partitioning the spatial variation of community composition data. Ecol. Monogr. 75: 435-450.

Levin, S.A. 1992. The problem of pattern and scale in ecology: the Robert H. MacArthur award lecture. Ecology 73: 1943-1967. 
Li, L., Z.L. Huang, W.H. Ye, H.L. Cao, S.G. Wei, Z.G. Wang, J.Y. Lian, I.F. Sun, K.P. Ma and F.L. He. 2009. Spatial distributions of tree species in a subtropical forest of China. Oikos 118: 495502 .

Lomolino, M.V. 2000. Ecology's most general, yet protean pattern: the species-area relationship. J. Biogeogr. 27: 17-26.

Loreau, M. 2000. Are communites saturated? On the relationship between $\alpha, \beta$ and $\gamma$ diversity. Ecol. Lett. 3: 73-76.

MacArthur, R., H. Recher and M.Cody. 1966. On the relation between habitat selection and species diversity. Am. Nat. 100: 319332.

MacArthur, R.H. and E.O. Wilson. 1967. The Theory of Island Biogeography. Princeton University Press, Princeton, NJ.

MacNally, R., E. Fleishman, L.P. Bulluck and C.J. Estrus. 2004. Comparative influence of spatial scale on beta diversity within regional assemblages of birds and butterflies. J. Biogeogr. 31: 917-929.

Martín, H. G. and N. Goldenfeld. 2006. On the origin and robustness of power-laws pecies-area relationships in ecology. PNAS 103: 10310-10315.

Palmer, M.W. and P.S. White. 1994. Scale dependence and the Species-Area Relationship. Am. Nat. 144: 717-740.

$\mathrm{R}$ Development Core Team. 2011. R: A language and environment for statistical computing. R Foundation for Statistical Computing, Vienna,Austria. ISBN 3-900051-07-0, URL http:// www.R-project.org/.

Rahbek, C. 2005. The role of spatial scale and the perception of largescale species-richness patterns. Ecol. Lett. 8: 224-239.

Ricotta, C. \& M. Marignani. 2007. Computing $\beta$-diversity with Rao's quadratic entropy: a change of perspective. Divers. Distrib. 13 237-241

Ricotta, C. 2008. Computing additive beta-diversity from presence and absence scores: a critique and alternative parameters. Theor. Popul. Biol. 73: 244-249.

Ricotta, C. 2010. On beta diversity decomposition: trouble shared is not trouble halved. Ecology 91: 1981-1983.

Ritz, C. and J.C. Streibig. 2008. Nonlinear Regression with $R$. Springer, New York.

Rosenzweig, M.C. 1995. Species Diversity in Space and Time. Cambridge University Press, Cambridge.

Sandel, B. and A.B. Smith. 2009. Scale as a lurking factor: incorporating scale-dependence in experimental ecology. Oikos 118 : 1284-1291.
Scheiner, S. M. 2003. Six types of species-area curves. Global Ecol. Biogeogr. 12: 441-447.

Tuomisto, H. 2010a. A diversity of beta diversities: straightening up a concept gone awry. Part 1. Defining beta diversity as a function of alpha and gamma diversity. Ecography 33: 2-22.

Tuomisto, H. 2010b. A diversity of beta diversities: straightening up a concept gone awry. Part 2. Quantifying beta diversity and related phenomena. Ecography 33: 23-45.

Turner, W.R. and Tjørve E. 2005.Scale-dependence in species-area relationships. Ecography 28: 721-730.

Veech, J.A. and T.O. Crist. 2010. Toward a unified view of diversity partitioning. Ecology 91: 1988-1992.

Vellend, M. 2001. Do commonly used indices of $\beta$-diversity measure species turnover? J. Veg. Sci. 12: 545-552.

Xiao, X., E.P. White, M.B. Hooten and S.L. Durham. 2011. On the use of logtransformation vs. nonlinear regression for analyzing biological power laws. Ecology 92: 1887-1894.

Whittaker, R.H. 1972. Evolution and measurement of species diversity. Taxon 21: 213-251.

Whittaker, R. J. and T. J. Matthews. 2014. The varied form of species-area relationships. J. Biogeogr. 41: 209-210.

Wiens, J.A. 1989. Spatial scaling in ecology. Funct. Ecol. 3: 385-397.

Wilson, M. V. and A. Shmida. 1984. Measuring beta diversity with presence-absence data. J. Ecol. 72: 1055-1064.

Zhang, Y.X., K.M. Ma, M. Anand and B.J. Fu. 2006. Do generalized scaling laws exist for species abundance distribution in mountains? Oikos 116: 81-88.

Received April 15, 2014

Revised June 9, August 13, 2014

Accepted September 3, 2014

\section{Electronic supplements}

Mathematical inference for beta diversity partitioned by multiplicative approach.

\section{Figures S1-S3.}

The file may be downloaded from www.akademiai.com. 